\title{
Amiodarone-Induced Thyrotoxicosis and Ventricular Arrhythmias: Case Report and Review of the Literature
}

\author{
Ashish Shah, MD \\ Thomas Jefferson University, ashish.shah@jefferson.edu
}

Follow this and additional works at: https://jdc.jefferson.edu/tmf

Part of the Endocrinology, Diabetes, and Metabolism Commons, and the Internal Medicine Commons Let us know how access to this document benefits you

\section{Recommended Citation}

Shah, MD, Ashish (2016) "Amiodarone-Induced Thyrotoxicosis and Ventricular Arrhythmias: Case Report and Review of the Literature," The Medicine Forum: Vol. 17 , Article 13.

DOI: https://doi.org/10.29046/TMF.017.1.014

Available at: https://jdc.jefferson.edu/tmf/vol17/iss1/13

This Article is brought to you for free and open access by the Jefferson Digital Commons. The Jefferson Digital Commons is a service of Thomas Jefferson University's Center for Teaching and Learning (CTL). The Commons is a showcase for Jefferson books and journals, peer-reviewed scholarly publications, unique historical collections from the University archives, and teaching tools. The Jefferson Digital Commons allows researchers and interested readers anywhere in the world to learn about and keep up to date with Jefferson scholarship. This article has been accepted for inclusion in The Medicine Forum by an authorized administrator of the Jefferson Digital Commons. For more information, please contact: JeffersonDigitalCommons@jefferson.edu. 


\title{
Amiodarone-Induced Thyrotoxicosis and Ventricular Arrhythmias: Case Report and Review of the Literature
}

\author{
Ashish Shah, MD
}

\section{INTRODUCTION}

Amiodarone is a common drug used to treat arrhythmias and its side effects are diverse. An important adverse effect is amiodarone-induced thyrotoxicosis (AIT), which can potentially lead to a pro-arrythmogenic state. There have been limited studies on the pathogenesis of how thyrotoxicosis increases the risk of ventricular arrhythmias, which causes providers to face a therapeutic challenge in patients who require this medication for the prevention of life threatening arrhythmias. This case report will present a patient with a left ventricular assist device (LVAD) who had recurrent ventricular arrhythmias on chronic amiodarone therapy in the setting of AIT.

\section{CASE PRESENTATION}

A 50 year old male with history of non-ischemic cardiomyopathy (EF 15\%) and an LVAD awaiting transplant presented to the hospital in ventricular fibrillation/torsades de pointes requiring electrical cardioversion. His other past medical history included hypertension, obstructive sleep apnea, hyperlipidemia, chronic kidney disease and ventricular tachycardia status post implantable cardioverter defibrillator (ICD) placement. The patient's ICD was removed a year prior to admission due to device endocarditis, MRSA/MSSA bacteremia, and epidural abscess/spinal osteodiscitis. Due to the history of ventricular tachyarrhythmias and removal of the ICD, he was on chronic amiodarone therapy $(200 \mathrm{mg}$ per day) for the past 1.5 years.

On admission he denied any symptoms of thyroid dysfunction such as weight changes, temperature sensitivity, recent fractures, fatigue/weakness, changes in bowel movements, hair loss, or changes in sleep. On physical exam, his vital signs were stable following cardioversion and he appeared clinically euthyroid without signs of thyroid associated ophthalmopathy (TAO), moist palms or dry skin, tremor, peripheral edema, or abnormal skin temperature. His thyroid exam was normal. Significant laboratory studies are shown in Table 1.

\section{DIFFERENTIAL DIAGNOSIS}

Based on the laboratory values, the differential includes thyroid diseases such as Graves' Disease, thyroiditis, toxic nodule or toxic multinodular goiter or AIT type 1 or type 2. The combination of radioactive iodine uptake (RAIU) and scan, the detection of autoantibodies, and color flow Doppler sonography can help narrow down this differential.

\section{OUTCOME AND FOLLOW-UP}

During the patient's hospital stay, he was continued on an increased dose of amiodarone therapy and a new ICD was placed successfully without complications. A thyroid ultrasound revealed a hypoechoic right thyroid nodule measuring $1.6 \times 0.9 \times 1.1 \mathrm{~cm}$ and normal thyroid size. A radioactive thyroid uptake and scan showed markedly reduced thyroid activity. An ultrasound guided biopsy of the thyroid nodule showed minimal blood flow through the nodule using Doppler evaluation. The FNA was performed to rule out malignancy in the setting of being on the transplant list and the pathology was a benign follicular nodule. Thyroid Stimulating Immunoglobulins were not elevated. He was diagnosed as having type 2 AIT. Upon discharge, the patient's amiodarone dose was

\begin{tabular}{|c|c|c|c|c|c|}
\hline & $\begin{array}{l}1 \text { month } \\
\text { after hospital } \\
\text { admission }\end{array}$ & $\begin{array}{l}\text { During } \\
\text { hospital } \\
\text { admission }\end{array}$ & $\begin{array}{l}2 \text { months } \\
\text { prior to } \\
\text { admission }\end{array}$ & $\begin{array}{c}1 \text { year prior to } \\
\text { admission ( } 6 \text { months on } \\
\text { amiodarone therapy) }\end{array}$ & $\begin{array}{l}2 \text { years prior to } \\
\text { admission (before } \\
\text { amiodarone therapy) }\end{array}$ \\
\hline $\begin{array}{l}\text { Thyroid Stimulating } \\
\text { Hormone (ulU/mL) } \\
\text { (normal 0.3-5) }\end{array}$ & 0.58 & $<0.02$ & $<0.02$ & 5.21 & 2.40 \\
\hline $\begin{array}{l}\text { Free T4 (ng/dL) } \\
\text { (normal } 0.7-1.7)\end{array}$ & 1.3 & 1.9 & 3.6 & 1.5 & Not checked \\
\hline $\begin{array}{l}\text { Free T3 (ng/dL) } \\
\text { (normal 2-4.2) }\end{array}$ & 1.9 & 3.8 & $\begin{array}{l}\text { Not } \\
\text { checked }\end{array}$ & Not checked & Not checked \\
\hline
\end{tabular}


The Medicine Forum, Vol. 17 [2016], Art. 13

\begin{tabular}{|l|c|c|}
\hline \multicolumn{2}{|c|}{ Table 2: Amiodarone-induced thyrotoxicosis classification } \\
\hline
\end{tabular}

increased to $400 \mathrm{mg}$ daily. The patient was followed as an outpatient and his thyroid function tests normalized within one month of hospitalization without requiring therapy. His thyroid function tests continue to be closely monitored and he is currently listed for heart transplant.

\section{DISCUSSION}

Amiodarone is an iodine rich drug that is used commonly in the treatment of both ventricular and atrial arrhythmias. Amiodarone affects multiple organ systems and can have varying effects on the thyroid as well. It can result in abnormalities in thyroid function tests as well as clinically apparent thyrotoxicosis or even hypothyroidism. Amiodarone is $37 \%$ iodine by weight and is structurally similar to T4.1. Taking $200 \mathrm{mg}$ of amiodarone daily releases approximately $7 \mathrm{mg}$ of iodide per day, which far exceeds the daily recommended optimal dose of iodine of 150ug1. Amiodarone and its metabolite, desethylamiodarone, have long half-lives of approximately 53 days and 61 days respectively. As a result of the increased iodine content, a very long half life, and systemic distribution into the adipose tissues, amiodarone can continue to cause clinical effects after cessation of therapy.

For patients who are on chronic amiodarone therapy and are clinically euthyroid, thyroid function tests typically display an increased serum T4 concentration and a decreased serum T3 concentration. Amiodarone inhibits the type 1-5'-deiodinase in peripheral tissues, which is responsible for the conversion of T4 to T31. Typically, patients will have serum T4 concentrations at the upper limit of normal and serum T3 concentrations within the low normal range. The TSH in these patients are usually normal however initially after starting amiodarone therapy the TSH can be elevated as was seen in our patient after he was on therapy for 6 months

AIT has an incidence of $14-18 \%$ of patients on chronic amiodarone therapy ${ }^{1}$. A significant risk factor for this adverse effect is taking a dose of $200 \mathrm{mg}$ daily ${ }^{1}$.
Thyrotoxicosis can occur at any point during amiodarone therapy, with an average length of treatment being three years before occurrence ${ }^{1}$. AlT has been classified into two types (type 1 AIT and type 2 AIT), although distinguishing between both types can be difficult.

In type 1 AIT, excessive thyroid hormone synthesis is induced by the iodine load associated with amiodarone therapy ${ }^{1}$. These patients typically have a prior thyroid abnormality such as diffuse or nodular goiter or latent Graves' disease and live in a geographic area that is slightly iodine-deficient ${ }^{1}$. Their thyroid gland does not respond to the iodine load which would normally decrease the synthesis of thyroid hormone, but it rather continues to uptake more iodine and synthesize more thyroid hormone

This type of AIT has increased RAIU and normal or slightly elevated serum interleukin 6 levels, a cytokine that indicates thyroid cell destruction, and thus there is no significant thyroid cell destruction in type 1 AlT $^{1}$. On ultrasound with color flow Doppler, there is an increase in color flow indicating hypervascularity as well as increased functioning of the thyroid

Patients with type 2 AlT typically have a normal thyroid gland prior to amiodarone therapy and do not have thyroid autoantibodies.1. They have very low RAIU and elevated serum interleukin 6 levels'. On ultrasound with color flow Doppler, there is typically less color flow in any area of the thyroid indicating decreased vascularity and decreased function of the thyroid gland ${ }^{1}$. These features of type 2 AIT infer that the pathophysiology involves thyroid cell destruction with evidence follicular damage ${ }^{1}$ The damaged follicles then release their preformed thyroid hormones leading to clinical symptoms of AIT. Table 2 summarizes the classification of AIT.

Treatment of type I AIT is primarily blocking organification of excess iodine and the synthesis of thyroid hormones? Higher doses of thionamides such as methimazole or propylthiouracil are utilized. Another drug used to block the excess iodine from entering the thyroid is potassium 
perchlorate (although not available in the US), which can be combined with the thionamides. Treatment of type 2 AIT is primarily with high dose steroids (40-60 mg/day) due to their anti-inflammatory and membrane stabilizing effects on the thyroid along with inhibition of $5^{\prime}$-deiodinase ${ }^{1}$. If a patient has features of both type 1 and type 2 AIT, a combination of thionamides/potassium perchlorate and steroids can be used. If these treatment options are not successful, the last options are radioactive iodine (for type 1) or total thyroidectomy (for both).

In our patient, his T4 was elevated in the setting of a suppressed TSH both two months prior to admission and on admission. He had a right thyroid nodule but no known autoantibodies or prior thyroid dysfunction. His RAIU was suppressed throughout the thyroid gland. Interleukin 6 levels were not obtained. On thyroid ultrasound, there was no increased color flow uptake. As a result, the patient was diagnosed with a likely mild case of type 2 AIT. Clinically, he had limited symptoms of thyrotoxicosis which may have been secondary to his previously prescribed beta-blocker (carvedilol).

The more interesting finding is that this patient had an episode of ventricular fibrillation/torsades de pointes in the setting of type 2 AIT. A recent study done in Japan on patients on chronic amiodarone therapy showed that recurrent sustained ventricular tachycardia was significantly higher with thyrotoxicosis than in the euthyroid state 4 . Holter monitoring of these patients with thyrotoxicosis actually showed that the heart rate along with the number of ventricular premature complexes significantly increased $^{4}$. One case report in Australia documented a patient on chronic amiodarone therapy for ventricular ectopy developing a coronary vasospasm and recurrent ventricular fibrillation in the setting of type $2 \mathrm{AlT}^{5}$. It is difficult to conclude whether our patient's type 2 AIT had any role in contributing to his ventricular fibrillation/ torsades de pointes episode prior to admission or whether this was the progression of his non-ischemic cardiomyopathy. It seems fair to conclude that triggers for his arrhythmia are multifactorial in nature. One recent study showed that the rate of major adverse cardiovascular events (cardiovascular mortality, myocardial infarction, stroke and heart failure, or ventricular arrhythmias requiring hospitalization) is 2.7 fold greater for those patients on long term amiodarone therapy who develop AIT than those who do not develop AIT, with ventricular arrhythmias being the most frequent type of event ${ }^{6}$. It has also been found that in the setting of AIT, severe left ventricular dysfunction is associated with increased mortality?
In conclusion, this case displays the effects of chronic amiodarone therapy on thyroid function. Clinicians must weigh the risks and benefits of using this anti-arrhythmic in the setting of a patient's underlying cardiomyopathy. Given this patient's history of ventricular arrhythmias, the benefit of being on amiodarone therapy greatly outweighs the risks of developing thyrotoxicosis. However after presenting to the hospital with episodes of ventricular fibrillation/torsades de pointes in the setting of type 2 AIT, one has to carefully follow the thyroid function tests to ensure biochemical normalization in order to minimize the risk for increased ventricular arrhythmia burden in addition to his worsening left ventricular ejection fraction. Overall, this is a unique case of an LVAD patient being bridged to transplant with recurrent ventricular arrhythmia on chronic amiodarone therapy who developed type 2 AIT. The management of these arrhythmias in the setting of AIT need to be further studied in order to balance the beneficial anti-arrhythmic properties of amiodarone with its harmful ability to cause thyrotoxicosis leading to a pro-arrythmogenic state.

\section{REFERENCES}

1. Martino, Enio. The Effects of Amiodarone on the Thyroid. Endocrine Reviews. 2001; 22(2): $240-254$

2. W Tsang, RL Houlden. Amiodarone-induced thyrotoxicosis: A review. Can J Cardiol 2009; 25(7):421-424

3. Narayana, Shashithej. Management of amiodarone-related thyroid problems. Therapeutic Advances in Endocrinology and Metabolism. 2011; 2(3):115-126.

4. Shiga, Tsuyoshi. Amiodarone-induced thyroid dysfunction and ventricular tachyarrhythmias during long-term therapy in Japan. Jpn Circ J. 2001; 65: 958-960.

5. Brooks, Matthew. Amiodarone-induced destructive thyroiditis associated with coronary artery vasospasm and recurrent ventricular fibrillation. Eur Thyroid J. 2013; 2:65-67.

6. Yiu, Kai-Hang. Amiodarone-Induced thyrotoxicosis is a predictor of adverse cardiovascular outcome. J Clin Endocrinol Metab. 2009; 94: 109-114.

7. O'Sullivan, Anthony. Amiodarone-induced thyrotoxicosis: left ventricular dysfunction is associated with increased mortality. European Journal of Endocrinology. 2006; 154:533-536. 